\title{
Irregularity of energy intake at meals: prospective associations with the metabolic syndrome in adults of the 1946 British birth cohort
}

\author{
Gerda K. Pot ${ }^{1 *}$, Rebecca Hardy ${ }^{2}$ and Alison M. Stephen ${ }^{3}$ \\ ${ }^{1}$ School of Medicine, Diabetes and Nutritional Sciences Division, King's College London, Franklin-Wilkins Building, \\ 150 Stamford Street, London SE1 9NH, UK \\ ${ }^{2}$ MRC Unit for Lifelong Health and Ageing, University College London (UCL), London, UK \\ ${ }^{3}$ Department of Nutritional Sciences, Faculty of Health and Medical Sciences, Surrey University, Surrey, UK
}

(Submitted 5 June 2015 - Final revision received 4 September 2015 - Accepted 5 October 2015- First published online 9 November 2015)

\section{Abstract}

Irregularity in eating patterns could be a potential cardiometabolic risk factor. We aimed to study the associations of irregular intake of energy at meals in relation to cardiometabolic risk factors 10 and 17 years later. Variability of energy intake data - derived from 5-d estimated diet diaries of cohort members of the National Survey for Health and Development collected at ages $36(n 1416)$, 43 ( $n$ 1505) and 53 years ( $n$ 1381) - was used as a measure for irregularity. Associations between meal irregularity scores with cardiometabolic risk factors measured 10 and 17 years later were investigated using linear mixed models and logistic regression models. The results showed that irregularity scores changed significantly over the years $(P<0.05)$. At age 36 years, subjects with a more irregular intake of energy at lunch (OR $1.42 ; 95 \%$ CI 1.05 , 1.91) and between meals (OR 1.35; $95 \%$ CI 1.01, 1.82) had an increased risk for the metabolic syndrome 17 years later; at lunch was also associated with an increased waist circumference (OR 1.58; $95 \% 1.27,1.96$ ) and TAG levels (OR 1.33; 95\% CI 1.02, 1.72). At age 43 years, subjects with a more irregular intake at breakfast had an increased risk of the metabolic syndrome 10 years later (OR 1.53; $95 \%$ CI 1.15, 2.04), as well as an increased BMI (OR 1.66; $95 \%$ CI 1.31, 2.10), waist circumference (OR 1.53; $95 \%$ CI 1.23, 1.90) and diastolic blood pressure (OR 1.42; $95 \%$ CI 1.13, 1.78). In conclusion, subjects with a more irregular intake of energy, mostly at breakfast and lunch, appeared to have an increased cardiometabolic risk 10 and 17 years later.

Key words: Irregular intake: Energy intake: Chronobiology: Cardiometabolic risks: National Survey of Health and Development: Prospective studies

From a clinical and public health nutrition perspective, the concept of the 'sex non-res naturales' (the six non-natural things) as described by Hippocrates (460-377 BC) and later used in Florence Nightingale's Notes on Nursing ${ }^{(1)}$ is still contemporary. The sex non-res naturales include the topic of food and drinks and emphasise the importance of regularity and rest. Regular or consistent meal patterns are thought to be beneficial for our health and are concepts common to many diets. However, the scientific evidence relating these types of meal patterns with health is limited, especially from studies that have detailed dietary assessment. We define irregularity of meals as food being consumed in varying amounts through the day and at different times from one day to the next, and we use this term interchangeably with inconsistent meal patterns. This implies a link with chronobiology, the study of biological rhythms, which are termed circadian rhythms if the cycle is one of $24 \mathrm{~h}$. Several physiologically and nutritionally relevant processes in the human body appear to have a circadian rhythm, including cortisol secretion, blood glucose metabolism and gastrointestinal tract motility ${ }^{(2)}$. In addition, energy metabolism and dietary intake are both under strong circadian control $^{(3)}$. It is known that dietary intake can act as a zeitgeber - that is, providing a stimulus for the setting or re-setting of a biological clock $^{(4,5)}$. Linking chronobiology with nutritional research was proposed in the late 1980s - for example, by Halberg in $1989^{(6,7)}$ with numerous in vitro studies. This was later followed-up by some research in shift workers ${ }^{(8-10)}$ and studies investigating the time of day of food intake in humans ${ }^{(11,12)}$. The discovery of circadian rhythmicity in adipose tissue genes in $2005^{(13,14)}$ further revived this area of study to consider not only 'what' people consume but also 'when' they normally consume it ${ }^{(15)}$.

Studying meal timing involves different aspects of time: clock time (i.e. time of day), frequency (i.e. events per time span) and regularity (i.e. similarity of events at specific times). Thus far, only two human intervention trials have provided evidence for the latter aspect of time - that is, regularity - and found that regular meal patterns could have beneficial effects on cardiometabolic risk factors ${ }^{(16,17)}$. Evidence from large observational

Abbreviation: NSHD, National Survey of Health and Development.

* Corresponding author: G. K. Pot, email Pot@kcl.ac.uk 
studies with detailed data collection methods such as food diaries is even scarcer. We previously investigated the association of irregular intake of energy during meals with cardiometabolic disease risk using data from the Medical Research Council's National Survey of Health and Development (NSHD) and showed that irregular intake of energy, especially at breakfast and between meals, was associated with a higher cardiometabolic disease risk ${ }^{(18)}$. However, these analyses were cross-sectional. NSHD is the world's longest-running birth cohort and has collected data including detailed dietary information at regular intervals in adulthood ${ }^{(19)}$. Therefore, this research focuses on how irregular intakes of energy at meals at two time points over almost two decades are associated with cardiometabolic disease risk factors 10 and 17 years later.

\section{Methods}

\section{Subjects}

NSHD, also known as the 1946 British birth cohort, is a longitudinal study based on a social class-stratified sample of 5632 singleton births that occurred within marriage in England, Scotland and Wales during 1 week in March $1946^{(20,21)}$. Data have been collected on this cohort on more than twenty occasions, including collection of dietary data when participants were aged 36 (1982), 43 (1989), 53 (1999) and 60-64 years (2006-2010). This study has been conducted according to the guidelines laid down in the Declaration of Helsinki. Ethics approval was obtained from the North Thames Multicentre Research Ethics Committee and written informed consent was obtained from all cohort members.

\section{Assessment of dietary intake}

Details of the dietary assessment methods in this cohort have been described in detail previously ${ }^{(18,19)}$. In brief, dietary data were collected over 5 consecutive days at ages 36, 43 and 53 years using a 5-d estimated diet diary. All food and drinks consumed both at home and away were recorded using household measures, and portion sizes were estimated using detailed guidance notes and photographs provided at the beginning of the diary ${ }^{(22)}$. Diet diaries were coded using nutrient databases that were time-appropriate for each of the different time periods ${ }^{(18,19)}$. Diet diaries were pre-structured into breakfast, lunch, evening meal and between meals, which consisted of combining first thing (which was the time slot after waking up and before breakfast), mid-morning, tea, late evening and extras. The meal slots for first thing, mid-morning, tea, late evening and extras were collapsed into one meal slot for 'between meals'. Diet diaries were also collected at age 60-64 years, but were not included in the present analyses as they were structured with different time slots.

\section{Scores for irregularity of energy intake}

Calculations of the meal irregularity scores have been described in our previous paper presenting the cross-sectional data analysis in this cohort at age 53 years ${ }^{(18)}$. The scores for irregular intake of energy and alcohol were calculated per individual as the energy intake per meal time slot compared with the individual 5-d mean energy intake of that meal time slot, and the deviation from the mean per meal time slot was calculated, which served as a measure of regularity for that time slot, with a lower score indicating a more regular meal pattern and a higher score indicating more irregularity.

Mean irregularity scores were calculated for each of the four time slots (breakfast, lunch, evening meal and between meals) and as daily total at ages 36, 43 and 53 years.

Energy intake in $\mathrm{kJ} / \mathrm{d}(\mathrm{kcal} / \mathrm{d})$ and per time slot was obtained from the diet diaries, and the percentage of estimated energy requirements (EAR) was calculated. A population approach was used: EAR was $10899 \mathrm{~kJ} / \mathrm{d}$ (2605 kcal/d) for men and $8699 \mathrm{~kJ} / \mathrm{d}$ $(2079 \mathrm{kcal} / \mathrm{d})$ for women for an average BMI of $22.5 \mathrm{~kg} / \mathrm{m}^{2}$ and an average physical activity level of $1.63^{(23)}$.

\section{Prevalence of cardiometabolic risk factors and covariates}

During home visits by trained research nurses when cohort members were aged 53 years, height and weight were measured according to standard protocols and subsequently BMI was calculated.

At age 53 years, waist circumference and blood pressure (BP) were also measured, and a non-fasting venous blood sample was obtained to measure levels of HDL-cholesterol, TAG and glycated $\mathrm{Hb}(\mathrm{HbA} 1 \mathrm{c})^{(24)}$. Measures from non-fasting blood samples have been found to be indicators of CVD risk when the preferred fasting blood samples are unavailable in large observational studies ${ }^{(25,26)}$. Blood samples were not collected at age 36 or 43 years.

The definition of the metabolic syndrome and its components were based on a modified version of the Adult Treatment Panel III (ATP-III) definition of the metabolic syndrome, when $\geq 3$ of the following criteria were present ${ }^{(27)}$ : waist circumference $\geq 102 \mathrm{~cm}$ in men and $\geq 88 \mathrm{~cm}$ in women; HDL-cholesterol $<1.036 \mathrm{mmol} / 1$ (40 mg/dl) in men and $<1.295 \mathrm{mmol} / 1(50 \mathrm{mg} / \mathrm{dl})$ in women or specific treatment for this lipid abnormality; TAG $\geq 1.7 \mathrm{mmol} / 1(150 \mathrm{mg} / \mathrm{dl})$ in men and women or specific treatment for this lipid abnormality; systolic BP $\geq 130 \mathrm{mmHg}$ or diastolic BP $\geq 85 \mathrm{mmHg}$ in men and women or use of anti-hypertensive medication; and HbA1c level in the top sex-specific quarter of the distribution $(>5.8 \%$ among men and women) or use of medication for diabetes. The latter was a modification of the ATP-III criteria as fasting blood glucose measures were not available for this population at that time point.

Cohort members who had completed 4 or more days of the diet diary and for from whom the metabolic syndrome could be derived were included in these analyses: for age 36 years, $n 1416$ participants; for age 43 years, $n 1505$ participants; and for age 53 years, $n 1381$ participants. A total of $n 1007$ participants had completed the food diaries at all three time points, as well as were having data available on the metabolic syndrome at age 53 years.

Potential confounding variables were behavioural factors that have previously been associated with the metabolic syndrome. Structured questionnaires at ages 36, 43 and 53 years were used 
to obtain information on demographic and socio-economic variables, including socio-economic position (occupational social class was used to represent socio-economic position: no job, professional, intermediate, skilled non-manual, skilled manual, partly skilled, unskilled and armed forces), marital status (married, single or divorced/widowed/separated), physical activity (categories: none, 1-4 times/month or $\geq 5$ times/month of sports or recreational activity) and smoking (current, ex-smoker or never smoked) ${ }^{(19)}$

\section{Statistical analyses}

The statistical analyses were split into the following two parts: (1) a description of the population and changes in meal irregularity scores with age, and (2) analysis of the relationship between irregularity scores at ages 36 and 43 years and the metabolic syndrome at age 53 years. Characteristics of the cohort members included in this study and irregularity scores per meal time slots were presented for each age group (36, 43 and 53 years) as mean values and standard deviations or as percentage for discrete variables.

\section{Changes in meal irregularity scores of energy intake. Linear}

mixed models were used to examine changes in irregularity scores of energy intake with age; this method uses all available data over follow-up, takes into account the fact that repeated measures on the same individual are correlated, and it can handle missing outcomes under the assumption that data are missing at random ${ }^{(28)}$. In these analyses, both the intercept and the slope were fitted as random effects, allowing cohort members to have different irregularity scores at baseline and different rates of change over follow-up. The models included terms for time (year of dietary data collection). An interaction between time and compliance was then added to examine whether there were differences in meal irregularity scores between survey members who completed dietary assessment at all three time points and those who provided data only at one or two different time points.

\section{Diet at 36 and 43 years of age in relation to cardiometa-} bolic risk factors at 53 years of age. The primary outcome was metabolic syndrome at age 53 years. Secondary outcomes were having one of the ATP-III metabolic syndrome components or an increased BMI $\left(\geq 25 \mathrm{~kg} / \mathrm{m}^{2}\right)$. BMI is not a component of the metabolic syndrome, but was included as it is an important cardiometabolic risk factor. Multiple logistic regression models were used to calculate the odds of having the metabolic syndrome at age 53 years per tertile of the irregularity score for each time slot as well as the daily total irregularity score. This was first carried out for meal irregularity scores at age 36 years and then for scores at age 43 years. Results of the crosssectional analyses at age 53 years have previously been reported $^{(18)}$.

All the models were adjusted for sex, SES, marital status, physical activity and smoking status as confounding variables (model 1). To test for linear trends across tertiles, median intake scores of the respective tertile were assigned to each individual, and the resulting variable was fitted as a continuous variable in the model.

For the primary outcome, a second model was fitted, which also adjusted for energy intake at the other meal time slots, and models including the daily total irregularity scores were also adjusted for EAR (model 2). In addition, to assess the possible impact of under- or over-reporting of energy intake, sensitivity analyses were carried out including plausible reporters only. Under-reporters and over-reporters were identified based on the methods described by McCrory et al. ${ }^{(29)}$ and using $2 \mathrm{SD}$ as cut-off points.

Data analysis was carried out using SPSS for MS Windows 21.0 (SPSS Inc.) and a $P$ value of $<0.05$ was considered to be statistically significant.

\section{Results}

Of the cohort members included in these analyses, about $53 \%$ were women, and this was similar at the three time points (Table 1). The percentage of married cohort members decreased with age (from $87 \%$ in 1982 to $80 \%$ in 1999), as did the percentage of cohort members who smoked (from $28 \%$ in 1982 to $18 \%$ in 1999). The percentage of cohort members working as professionals remained fairly stable, and the percentage of cohort members who were physically active was approximately $37 \%$ at ages 36 and 53 years but was somewhat lower at age 43 years (28\%). Dietary intakes changed with age in this cohort. Most striking were the decreases in reported energy intake and fat intake being highest at age 43 years $(\mathrm{g} / \mathrm{d})$. Energy intakes at the evening meal decreased over time while those between meals increased. Energy intakes at breakfast and lunch remained fairly stable.

\section{Changes in meal irregularity scores}

For energy intake between meals, irregularity decreased with increasing age $(P<0.05)$ (Fig. 1). For meal irregularity at breakfast, lunch and for the daily total, a decrease was observed at the most recent age, whereas for the evening meal no linear trends were observed in irregularity (Fig. 1). These differences remained statistically significant after adjusting for covariates (model 1) as well as adding the year by compliance (data not shown). The percentage of plausible reporters increased over time, with $65 \%$ at age 36 years to $85 \%$ at age 53 years. At all ages, the percentage of plausible reporters decreased with increasing irregularity scores (Table 1).

\section{Diet at 36 and 43 years of age in relation to cardiometabolic risk factors at 53 years of age}

At age 36 years, a more irregular intake of energy at lunch was associated with increased odds of having the metabolic syndrome 17 years later (Table 2; model 1) (OR 1.42 for highest $v$. lowest irregularity group, $95 \%$ CI 1.05, 1.91). A similar association was observed with irregular intake of energy between meals. These associations remained after further adjustment for energy intake (Table 2; model 2). In analyses including only plausible 
Table 1. Characteristics of the National Survey of Health and Development cohort members at ages 36 (1982), 43 (1989) and 53 years (1999) (Numbers and percentages; mean values and standard deviations)

\begin{tabular}{|c|c|c|c|c|c|c|}
\hline & 36 Years & & 43 Years & & 53 Years & \\
\hline & $\%$ & $n 1416$ & $\%$ & n 1505 & $\%$ & $n 1381$ \\
\hline Sex (\% female) & 53 & & 53 & & 54 & \\
\hline Marital status (\% married) & 87 & & 82 & & 80 & \\
\hline Smoking (\% current) & 28 & & 24 & & 18 & \\
\hline SES (\% professional) & $7 \cdot 7$ & & 6.8 & & $8 \cdot 2$ & \\
\hline Physical activity ( $\% \geq 5$ times/month) & 39 & & 28 & & 36 & \\
\hline Plausible reporters (\%) & 65 & & 71 & & 85 & \\
\hline \multirow[t]{2}{*}{ Plausible reporters per tertile of daily irregularity score $(\% \mathrm{~T} 1 / \mathrm{T} 2 / \mathrm{T} 3)$} & $73 / 69 / 65$ & & $77 / 74 / 72$ & & $89 / 89 / 80$ & \\
\hline & Mean & SD & Mean & SD & Mean & SD \\
\hline BMI $\left(\mathrm{kg} / \mathrm{m}^{2}\right)$ & $23 \cdot 8$ & $3 \cdot 3$ & $24 \cdot 8$ & $3 \cdot 6$ & $26 \cdot 7$ & $4 \cdot 3$ \\
\hline Waist circumference (cm) & $82 \cdot 0$ & 11.6 & $82 \cdot 7$ & 11.9 & 89.7 & 27.3 \\
\hline Hip circumference $(\mathrm{cm})$ & NA & & 99.7 & 78.5 & $106 \cdot 1$ & $42 \cdot 0$ \\
\hline Total energy intake $(\mathrm{kJ} / \mathrm{d})$ & 8560 & 2665 & 9029 & 2602 & 8293 & 2109 \\
\hline Total energy intake $(\mathrm{kcal} / \mathrm{d})$ & 2046 & 637 & 2158 & 622 & 1982 & 504 \\
\hline Fat intake $(\mathrm{g} / \mathrm{d})$ & $73 \cdot 2$ & 19.5 & 78.5 & $20 \cdot 6$ & $77 \cdot 8$ & $18 \cdot 0$ \\
\hline Fat intake (\% energy) & 39.4 & $5 \cdot 0$ & $39 \cdot 1$ & 5.5 & $34 \cdot 3$ & $6 \cdot 1$ \\
\hline Protein intake $(\mathrm{g} / \mathrm{d})$ & $89 \cdot 6$ & $29 \cdot 9$ & 94.5 & 31.9 & $76 \cdot 2$ & $25 \cdot 4$ \\
\hline Protein intake (\% energy) & $14 \cdot 8$ & $3 \cdot 1$ & 14.9 & $2 \cdot 7$ & $16 \cdot 0$ & $2 \cdot 8$ \\
\hline $\mathrm{CHO}$ intake $(\mathrm{g} / \mathrm{d})$ & $225 \cdot 6$ & $78 \cdot 0$ & $238 \cdot 1$ & $74 \cdot 2$ & $233 \cdot 6$ & $63 \cdot 3$ \\
\hline $\mathrm{CHO}$ intake (\% energy) & $41 \cdot 3$ & $5 \cdot 9$ & 41.5 & $6 \cdot 0$ & 44.6 & $7 \cdot 2$ \\
\hline Alcohol intake (g/d) & $13 \cdot 9$ & $20 \cdot 4$ & $13 \cdot 7$ & $18 \cdot 9$ & $15 \cdot 9$ & $21 \cdot 3$ \\
\hline \multicolumn{7}{|l|}{ Average energy intake at } \\
\hline Breakfast (kJ) & 1213 & 710 & 1192 & 711 & 1174 & 617 \\
\hline Breakfast (kcal) & 290 & 170 & 285 & 170 & 280 & 148 \\
\hline Lunch (kJ) & 2395 & 985 & 2504 & 1015 & 2227 & 872 \\
\hline Lunch (kcal) & 572 & 235 & 598 & 242 & 532 & 208 \\
\hline Evening meal (kJ) & 3469 & 1334 & 3242 & 1154 & 3014 & 1020 \\
\hline Evening meal (kcal) & 829 & 319 & 775 & 276 & 720 & 244 \\
\hline Between meals $(\mathrm{kJ})$ & 1419 & 1094 & 2035 & 1344 & 1878 & 1219 \\
\hline Between meals (kcal) & 339 & 261 & 486 & 321 & 449 & 291 \\
\hline
\end{tabular}

$\mathrm{T}$, tertile; $\mathrm{CHO}$, carbohydrate.

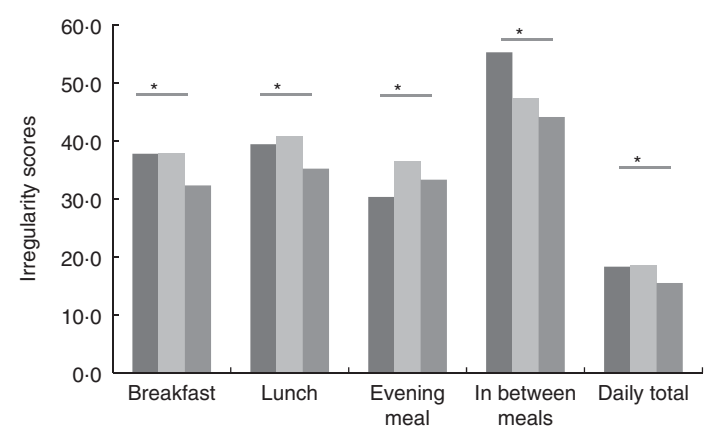

Fig. 1. Meal irregularity scores per meal time slot presented for the different years, ages 36,43 and 53 years $\left({ }^{\star} P<0.05\right.$ linear mixed models testing changes in irregularity scores with age). $\square, 36$ years $(n 2099) ;, 43$ years ( $n$ 2158);, 53 years $(n$ 1768).

reporters ( $65 \%$ of the main analytic sample), associations with irregular intake between meals remained; however, the association with irregular intake of energy at lunch was reduced and was no longer statistically significant (Table 2; model 1 plausible).

For the components of the metabolic syndrome, most statistically significant relationships with irregularity scores were found for overweight, either based on BMI or waist circumference, with more irregularity in energy intake at lunch, evening meal and daily total being associated with overweight 17 years later (Table 3). Moreover, having a more irregular energy intake at lunch at age 36 years was associated with increased TAG levels at age 53 years, and a more irregular daily total energy at age 36 years was inversely associated with decreased HDLcholesterol levels at age 53 years. For the other components of the metabolic syndrome at age 53 years, no statistically significant associations were found with irregularity of energy intake at age 36 years (Table 3).

At age 43 years, a more irregular intake of energy at breakfast was associated with increased odds of having the metabolic syndrome 10 years later (Table 2; model 1); this association also remained after further adjusting for energy intake (Table 2; model 2). Sensitivity analyses including plausible reporters only showed that this association was attenuated and was no longer statistically significant (Table 2 ; model 1 plausible).

For the components of the metabolic syndrome, a more irregular intake of energy at breakfast was associated with subsequent increased waist circumference 10 years later (Table 3). It was also found that individuals with an irregular intake of energy at breakfast, lunch and between meals had a higher chance of having a BMI $\geq 25 \mathrm{~kg} / \mathrm{m}^{2} 10$ years later. Moreover, a more irregular intake of energy at breakfast and lunch was associated with increased odds of having high diastolic BP 10 years later. 
Changes in irregularity of energy intake

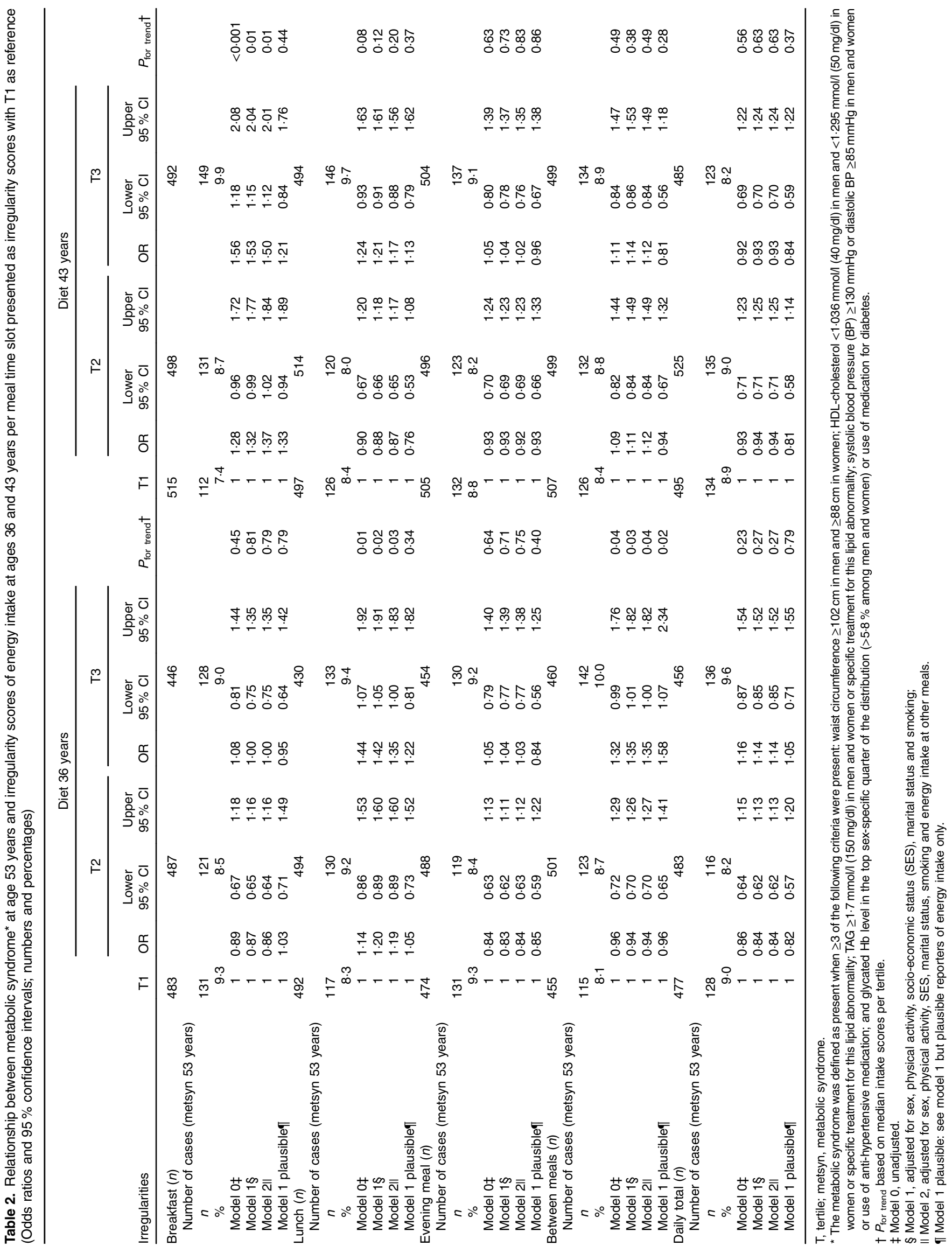




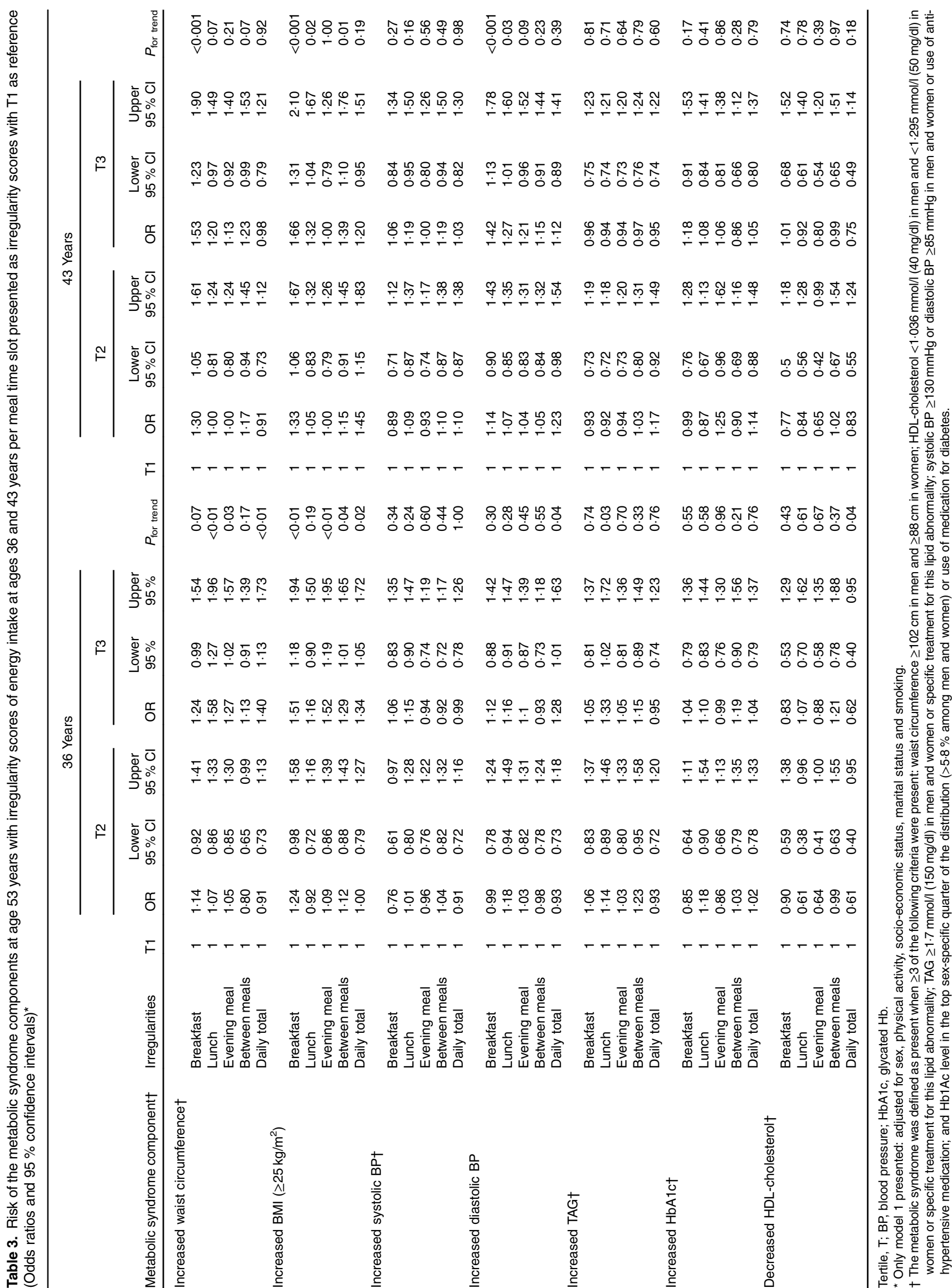




\section{Discussion}

The results of this prospective analysis showed that irregularity of energy intakes at breakfast, lunch, evening meal, between meals and daily total changed over the 17 years of follow-up in mid-life. Irregular intake of energy, both at lunch and breakfast, was associated with an increased risk of the metabolic syndrome, or its components, 10 and 17 years later. However, these associations were not always consistent for irregularity of energy intake at the two different ages. Irregular intake of energy at lunch and between meals at age 36 years was associated with an increased risk of the metabolic syndrome 17 years later, whereas at age 43 years it was irregular intake at breakfast that was associated with the metabolic syndrome 10 years later. Irregular intake of energy at lunch at 36 years of age and irregular intake at breakfast at 43 years of age were associated with high BMI at 53 years of age.

This investigation follows cross-sectional analyses in the same cohort at age 53 years, showing that cohort members with a more irregular intake of energy, especially at breakfast and between meals, had increased cardiometabolic risk ${ }^{(18)}$. This is one of the only nutritional epidemiological studies to date to include detailed dietary data to investigate the chronobiological effects of energy intake on cardiometabolic risk factors.

In this cohort, most scores for irregularity of energy intake decreased over the 17-year follow-up time, with the greatest drop in meal irregularity scores for energy intake between meals. A possible explanation for this decrease may be related to the fact that snacking behaviour has become more regular and more consistent in this cohort. A recent study of the food consumption patterns in the same cohort showed that consumption of sweet cereals, including biscuits, puddings, pastries, buns and pies, and confectionery had gone down from age 36 to $60-64$ years ${ }^{(30)}$. This, however, is not in line with other studies on healthy adults ${ }^{(31)}$ and children ${ }^{(32)}$, showing that snacking behaviour has increased, alongside an increased number of meals eaten away from home and meal skipping $^{(32,33)}$. It may be that the reduction in variability of snacking behaviour seen here is due to ageing, where lifestyle in general has become more regular with increasing age, as it has also been observed in other longitudinal studies $^{(34,35)}$. Previous studies have demonstrated that the dietary intake of this cohort was similar to the UK population of the same age at the time of data collection ${ }^{(36)}$, suggesting that similar trends would be observed in the general UK population of the same age.

It could be hypothesised that an increased BMI and waist circumference could be a mediating factor between the association of irregular intake of energy at meals and cardiometabolic disease risk. Therefore, in these analyses, we also studied associations between meal irregularity and the components of the metabolic syndrome, as well as high BMI. Most significant associations of irregularity of energy intake at ages 36 or 43 years with subsequent components of the metabolic syndrome 10 and 17 years later were found for overweight, either based on an increased waist circumference or BMI, supporting the hypothesis that the effects of meal irregularity on cardiometabolic disease risk could be mediated through obesity.
Other suggested underlying mechanisms of how changes in circadian rhythms leading to chronodisruption lead to an increased cardiometabolic risk could further be mediated through effects on glucose metabolism and other gastrointestinal routes. It has been shown that regulating and keeping the number of meals per $\mathrm{d}$ constant could minimise fluctuations in insulin concentration and plasma glucose levels ${ }^{(23,24)}$. However, measures of neither insulin nor glucose were available in NSHD at age 53 years, but HbA1c levels were analysed as a more long-term marker of glucose status. However, no associations were observed between increased HbA1c levels and irregularity scores. It may also be that more regular eating can induce more stable and constant plasma levels of intestinal satiety hormones such as glucagon-like peptide-1, cholecystokinin and peptide $\mathrm{YY}^{(24)}$. In addition, melatonin plays an important role in circadian rhythms, and is in part responsible for the establishment of an adequate energy balance. Reduction in melatonin concentrations, such as during ageing, shift-work or other chronodisruptive patterns, induces insulin resistance and glucose intolerance ${ }^{(37)}$. The studies by Farshchi et al. ${ }^{(16)}$ showed that having a more regular meal frequency led to improvement in peak insulin concentrations and AUC of insulin with a more regular meal frequency in healthy lean women. In healthy obese women, they also found that a more regular meal frequency led to lower energy intake and lower fasting total and HDL-cholesterol levels; however, no effect on glucose and insulin values were observed ${ }^{(17)}$. Most of this evidence relates to the effects of meal frequency, and more investigations into the effects of meal irregularity are warranted, including consumption of foods in varying amounts through the day and at different times from one day to the next.

It is important to consider that we studied the link between meal irregularity and subsequent prevalence of the metabolic syndrome, and it is possible that some cohort members may have already developed the metabolic syndrome by age 43 years (data not collected), which could have influenced the findings. Another possible bias may be due to underreporting of dietary intake. As previously also shown at age 53 years $^{(18)}$, the percentage of plausible reporters was higher for those who reported a more regular intake of energy. It was observed that some statistically significant findings became non-significant when only plausible reporters of energy intake were included, although this was partly due to the decreased sample size and loss of statistical power. It is conceivable that those with a more irregular intake of energy would be more likely to under-report their dietary intake, and this may account for the general weakening of the associations observed in the restricted sample.

In a prospective cohort study such as the NSHD, sample attrition is unavoidable. It is possible that this may result in bias if the healthier and more health-conscious cohort members are more likely to remain in the study. However, when including the year by compliance interaction term in the linear mixed models, changes in irregular intake over time remained statistically significant, suggesting that the results were not affected by not including those who provided detailed dietary data at the three time points. Moreover, the sample at age 53 years has been shown to be representative in many 
characteristics of the general population of the same age when compared with Census data ${ }^{(20)}$.

In a prospective study such as the NSHD, the power is constrained by the original sample size. It is possible that our analyses were lacking in the statistical power required to detect relatively small effects and therefore that some of our null findings were type-2 errors.

A major strength of this investigation was the use of detailed dietary information based on 5-d estimated diet diaries, which are currently accepted as the 'gold standard' to estimate dietary intake and in particular energy intake ${ }^{(34)}$. Moreover, the same method for detailed dietary assessment was used at multiple time points, making prospective investigations into meal irregularity possible in a relatively large sample, generating one of the first studies of its kind. In addition, to study regularity of meals, the dietary assessment method of multiple-day food diaries seems to be the best available method, as other dietary assessment methods such as FFQ would not be able to capture this level of details in terms of energy intake per meal, and thus would not provide a reliable and valid measure of energy intake.

Overall, this prospective analysis showed some evidence that greater irregularity of energy intake, especially at lunch and breakfast, was significantly associated with the risk of metabolic syndrome up to 17 years later. This study on the chronobiological effects of nutrition on cardiometabolic risk factors warrants further investigation, as it could potentially be of benefit for those at an increased cardiometabolic risk. If it can be demonstrated that consuming meals at regular times could reduce cardiometabolic risk, it could provide a useful public health strategy in tackling the rise in numbers of cardiometabolic disease currently prevalent.

\section{Acknowledgements}

The authors are indebted to all the members who took part in the NSHD.

This work was supported by the Medical Research Council (grant numbers U1200632239, U123092720). The Medical Research Council had no role in the design, analysis or writing of this article.

G. K. P. and A. M. S. designed the research; G. K. P. conducted the research and analysed the data; G. K. P., R. H. and A. M. S wrote the paper.

The authors have no conflicts of interest to declare.

\section{References}

1. Nightingale F (1860) Notes on Nursing. New York: D. Appleton and Company.

2. Haus E \& Smolensky M (2006) Biological clocks and shift work: circadian dysregulation and potential long-term effects. Cancer Causes Control 17, 489-500.

3. Drapeau V \& Gallant AR (2012) Homeostatic and circadian control of food intake: clinical strategies to prevent overconsumption. Curr Obes Rep 2, 93-103.

4. Stephan FK (2002) The 'other' circadian system: food as a zeitgeber. J Biol Rhythms 17, 284-292.
5. Scheer FA, Morris CJ \& Shea SA (2013) The internal circadian clock increases hunger and appetite in the evening independent of food intake and other behaviors. Obesity (Silver Spring) 21, 421-423.

6. Halberg F (1989) Some aspects of the chronobiology of nutrition: more work is needed on 'when to eat'. J Nutr 119, 333-343.

7. Kant AK, Schatzkin A \& Ballard-Barbash R (1997) Evening eating and subsequent long-term weight change in a national cohort. Int J Obes Relat Metab Disord 21, 407-412.

8. De Assis MAA, Kupek E, Nahas MV., et al. (2003) Food intake and circadian rhythms in shift workers with a high workload. Appetite 40, 175-183.

9. Lennernäs M, Hambraeus L \& Akerstedt T (1995) Shift related dietary intake in day and shift workers. Appetite 25, 253-265.

10. Pasqua IC \& Moreno CRC (2004) The nutritional status and eating habits of shift workers: a chronobiological approach. Chronobiol Int 21, 949-960.

11. De Castro JM (2004) The time of day of food intake influences overall intake in humans. J Nutr 134, 104-111.

12. De Castro JM (2009) When, how much and what foods are eaten are related to total daily food intake. Br J Nutr $\mathbf{1 0 2}$, 1228-1237.

13. Aoyagi T, Shimba S \& Tezuka M (2005) Characteristics of circadian gene expressions in mice white adipose tissue and 3T3-L1 adipocytes. $J$ Health Sci 51, 21-32.

14. Ptitsyn AA, Zvonic S, Conrad SA, et al. (2006) Circadian clocks are resounding in peripheral tissues. PLoS Comput Biol 2, e16.

15. Garaulet M \& Madrid JA (2010) Chronobiological aspects of nutrition, metabolic syndrome and obesity. Adv Drug Deliv Rev 62, 967-978.

16. Farshchi HR, Taylor MA \& Macdonald IA (2004) Regular meal frequency creates more appropriate insulin sensitivity and lipid profiles compared with irregular meal frequency in healthy lean women. Eur J Clin Nutr 58, 1071-1077.

17. Farshchi HR, Taylor MA \& Macdonald IA (2005) Beneficial metabolic effects of regular meal frequency on dietary thermogenesis, insulin sensitivity, and fasting lipid profiles in healthy obese women. Am J Clin Nutr 81, 16-24.

18. Pot GK, Hardy R \& Stephen AM (2014) Irregular consumption of energy intake in meals is associated with a higher cardiometabolic risk in adults of a British birth cohort. Int J Obes $\mathbf{3 8}$, $1518-1524$.

19. Kuh D, Pierce M, Adams J, et al. (2011) Cohort profile: updating the cohort profile for the MRC National Survey of Health and Development: a new clinic-based data collection for ageing research. Int J Epidemiol 40, e1-e9.

20. Wadsworth M, Kuh D, Richards M, et al. (2006) Cohort profile: the 1946 National Birth Cohort (MRC National Survey of Health and Development). Int J Epidemiol 35, 49-54.

21. Prynne CJ, Wagemakers JJ, Stephen AM, et al. (2009) Meat consumption after disaggregation of meat dishes in a cohort of British adults in 1989 and 1999 in relation to diet quality. EurJ Clin Nutr 63, 660-666.

22. Prynne CJ, Paul AA, Mishra GD, et al. (2005) Changes in intake of key nutrients over 17 years during adult life of a British birth cohort. Br J Nutr 94, 368-376.

23. Scientific Advisory Committee on Nutrition (2011) Dietary Recommendations for Energy. London: Scientific Advisory Committee on Nutrition.

24. Richards M, Black S, Mishra G, et al. (2009) IQ in childhood and the metabolic syndrome in middle age: extended follow-up of the 1946 British Birth Cohort study. Intelligence 37, 567-572.

25. Stalenhoef AF \& de Graaf J (2008) Association of fasting and nonfasting serum triglycerides with cardiovascular disease and 
the role of remnant-like lipoproteins and small dense LDL. Curr Opin Lipidol 19, 355-361.

26. Nordestgaard BG, Langsted A \& Freiberg JJ (2009) Nonfasting hyperlipidemia and cardiovascular disease. Curr Drug Targets 10, 328-335.

27. Grundy SM, Cleeman JI, Daniels SR, et al. (2005) Diagnosis and management of the metabolic syndrome. An American Heart Association/National Heart, Lung, and Blood Institute Scientific Statement. Executive summary. Cardiol Rev 13, 322-327.

28. Twisk J (2013) Applied Longitudinal Data Analysis for Epidemiology. A Practical Guide, 2nd ed. New York: Cambridge University Press.

29. McCrory MA, Hajduk CL \& Roberts SB (2002) Procedures for screening out inaccurate reports of dietary energy intake. Public Heal Nutr 5, 873-882.

30. Pot GK, Prynne CJ, Almoosawi S, et al. (2015) Trends in food consumption over 30 years: evidence from a British birth cohort. Eur J Clin Nutr 69, 817-823.

31. McCrory MA \& Campbell WW (2010) Effects of eating frequency, snacking, and breakfast skipping on energy regulation: symposium overview. J Nutr 141, 144-147.
32. Nicklas TA, Baranowski T, Cullen KW, et al. (2001) Eating patterns, dietary quality and obesity. J Am Coll Nutr 20, 599-608.

33. McCrory MA, Fuss PJ, Saltzman E, et al. (2000) Dietary determinants of energy intake and weight regulation in healthy adults. J Nutr 130, 276S-279S

34. Paffenbarger RS, Kampert JB, Lee IM, et al. (1994) Changes in physical activity and other lifeway patterns influencing longevity. Med Sci Sports Exerc 26, 857-865.

35. Hubert HB, Bloch DA, Oehlert JW, et al. (2002) Lifestyle habits and compression of morbidity. J Gerontol A Biol Sci Med Sci 57, M347-M351.

36. Bates B, Lennox A, Prentice A, et al. (2014) National Diet and Nutrition Survey: results from years 1 to 4 (combined) of the rolling programme for 2008 and 2009 to 2011 and 2012. London: Public Health England Food Standards Agency. https://www.gov.uk/government/statistics/national-diet-andnutrition-survey-results-from-years-1-to-4-combined-of-therolling-programme-for-2008-and-2009-to-2011-and-2012 (accessed September 2014).

37. Allison KC, Goel N \& Ahima RS (2013) Delayed timing of eating: impact on weight and metabolism. Curr Obes Rep 3, 91-100. 